\title{
Analysis of Side-Lap Effect and Characterization of Understory Vegetation Using Full-Waveform ALS +
}

\author{
Pablo Crespo-Peremarch * and Luis Ángel Ruiz \\ Geo-Environmental Cartography and Remote Sensing Group (CGAT), Universitat Politècnica de València, \\ Camí de Vera s/n, 46022 València, Spain \\ * Correspondence: pabcrepe@cgf.upv.es \\ † Presented at the II Congress in Geomatics Engineering, Madrid, Spain, 26-27 June 2019.
}

Published: 12 July 2019

\begin{abstract}
Airborne full-waveform LiDAR (ALSFw) is able to register forest structure properties, essential for fire prevention, in more detail than airborne discrete LiDAR (ALSD). However, few studies have analyzed ALSFw methodological parameters (i.e., voxel size and assignation value) due to the complexity and lack of processing tools. In this paper we analyze the influence of the pulse density and ALSFw methodological parameters on the ALSFw metrics, as well as the characterization of understory vegetation through ALSFw. Results show that the influence of pulse density on ALSFW metrics may be modelled and the differences reduced by modifying ALSFw methodological parameters. Additionally, the potential of ALSFw for characterizing the mean height $\left(\mathrm{R}^{2}=0.949\right)$ and volume $\left(R^{2}=0.951\right)$ of the understory vegetation was also proved.
\end{abstract}

Keywords: airborne laser scanning; LiDAR; terrestrial laser scanning (TLS); pulse density; voxel size; forest structure; metrics

\section{Introduction}

Forest structure is a key factor for preventing wildfires [1]. In some forest types (e.g., Mediterranean forests) the gap between the understory and overstory vegetation plays an important role in the generation of severe wildfires (i.e., crown fires).

Traditional techniques to characterize forest structure have a high cost and are time consuming. The use of remote sensing techniques facilitates this task, providing critical information over large areas. Among remote sensing techniques, LiDAR (Light Detection and Ranging) consists of laser pulses emitted from a sensor and backscattered resulting in a 3D point cloud. More specifically, airborne full-waveform LiDAR (ALSFw) systems register the whole signal instead of a discrete point cloud. This signal provides more information about the different vertical layers of the vegetation (i.e., forest structure) [2].

The amount of data registered is highly increased when ALSFw is used instead of traditional discrete LiDAR (ALSD). One of the most extended methods for reducing ALSFw data and processing metrics for further analyses is voxelization. It consists of clustering ALSFw into voxels (i.e., threedimensional pixels) and assigning them a single value (e.g., the maximum, mean, etc., of all the data within the voxel). However, ALSFw has not been widely used yet due to the lack of processing tools and the amount of data to be processed [3]. The analysis of methodological parameters such as voxel size and assignation value have poorly been carried out [3], and they are fundamental for the practical use of these data and to avoid unsuitable effects such as side-lap.

On the other hand, registration of the forest vertical structure by ALSD is not uniform along the vertical axis, being the lower strata poorly registered due to the occlusion beneath the canopy [4]. ALSFw has showed capacity to detect the understory vegetation with better accuracy than ALSD [5]. 
Nevertheless, given the mentioned lack of processing tools and the volume of data, few studies have proved the capacity of ALSFw for registering the understory vegetation.

The aim of this paper is to assess (i) side-lap effect varying the pulse density and the ALSFw methodological parameters (i.e., voxel size and assignation value) in a temperate US Pacific Northwest forest; and (ii) the characterization of the understory vegetation through ALSFw, and using Terrestrial Laser Scanning (TLS) data as reference in a Mediterranean forest.

\section{Methods}

\subsection{Study Areas}

The current research was carried out in two study areas. Study area 1 [3] is located in the US Pacific Northwest, in Oregon. It is dominated by Douglas-fir (Pseudotsuga menziesii) with an infrequent presence of red cedar (Thuja plicata), western hemlock (Tsuga heterophylla), grand fir (Abies grandis), bigleaf maple (Acer macrophyllum) and red alder (Alnus rubra). Study area 2 [4] is located in the Natural Park of Espadán, in the Mediterranean Spanish province of Castellón. The dominant species are Aleppo pine (Pinus halepensis), maritime pine (Pinus pinaster) and cork oak (Quercus suber).

\subsection{Data}

ALSFw data were used for both study areas, while TLS was only used for study area 2. ALSFW data from study area 1 were registered in July 2010 by a Leica ALS60 with a pulse density of 10.4 pulses $\mathrm{m}^{-2}$ on average. Data were recorded with a footprint size of $0.25 \mathrm{~m}$ and a temporal sample spacing of $0.3 \mathrm{~m}$. ALSFw data from study area 2 were acquired in September 2015 by a LiteMapper 6800 whose average pulse density was 14 pulses $\mathrm{m}^{-2}$. Data were with a footprint size of $0.24 \mathrm{~m}$ and a pulse density of $0.15 \mathrm{~m}$.

Additionally, TLS data were used in study area 2 as reference for the characterization of the understory vegetation. TLS data were acquired in September and October 2015 with a FARO FOCUS 3D 120. Each plot was registered from nine scans (following the distribution described in [4]).

On the other hand, in study area 1 a total number of 30 squared-shape plots, with an area of $804.25 \mathrm{~m}^{2}$, were manually selected where pulse density was at least 16 pulses $\mathrm{m}^{-2}$ to test a larger range of density reductions. In study area 2, a total of 21 circular plots with a radius of $15 \mathrm{~m}$ were registered by ALSFw and TLS. These plots were selected to have a representative sample of the different dominant species and understory presence.

\subsection{ALSFW Metric Variation According to Pulse Density and Full-Waveform Parameters}

Once plots were selected for study area 1, pulse density from ALSFw within each plot was reduced from 16 pulses $\mathrm{m}^{-2}$ to 2 pulses $\mathrm{m}^{-2}$ with an interval of 1 pulse $\mathrm{m}^{-2}$. As a result, different data sets of ALSFw were obtained for the different pulse densities and plots. Afterwards, three ALSFW metrics (HOME: height of median energy, NP: number of peaks and RWE: return waveform energy), described and extracted following [6], were extracted for each combination of pulse density, voxel size (from $0.25 \mathrm{~m}$ to $1.55 \mathrm{~m}$ with an interval of $0.10 \mathrm{~m}$ ) and assignation value (maximum, mean, median, percentiles 90 and 95).

Once ALSFw metrics were extracted, their values were analyzed as a function of the pulse density. In this way, a negative exponential distribution [3] was fitted to the representation of the ALSFw metric values in the $Y$ axis and the pulse density in the $X$ axis. Different representations and fits were done for the different plots, and combination of voxel sizes and assignation values. This distribution increases steeply and then becomes stable at a given point, which in this case is a given pulse density. As each combination of voxel size and assignation value may be tested in 30 plots, the overall results for each combination were computed as the average of the 30 plots. Results were assessed by leave-one-out cross validation. 


\subsection{Characterization of Understory Vegetation}

On the other hand, ALSFw data from study area 2 was used for the characterization of the understory vegetation using TLS data as reference. In this case, a wider set of ALSFw metrics [4] were extracted from the 21 plots. These metrics were used in linear regression models as independent variables to estimate understory mean height and volume extracted from TLS data. Firstly, a model with a maximum of three metrics and the minimum Akaike Information Criterion [7] was selected for each variable to be estimated. Next, these models were assessed using the coefficient of determination $\left(\mathrm{R}^{2}\right)$, root mean square error (RMSE), normalized RMSE (nRMSE) and coefficient of variation $(\mathrm{CV})$.

\section{Results}

Figure 1 shows the pulse density at which the ALSFw metrics become stable (henceforth referred to as SP from sill point). This value is retrieved from the negative exponential distribution. SP values decrease as voxel size increases. Additionally, SP values decrease more steeply for the mean and median assignation of RWE, while the behavior for the different assignation values is more similar for HOME and NP. Moreover, SP values from HOME are lower compared to the others.

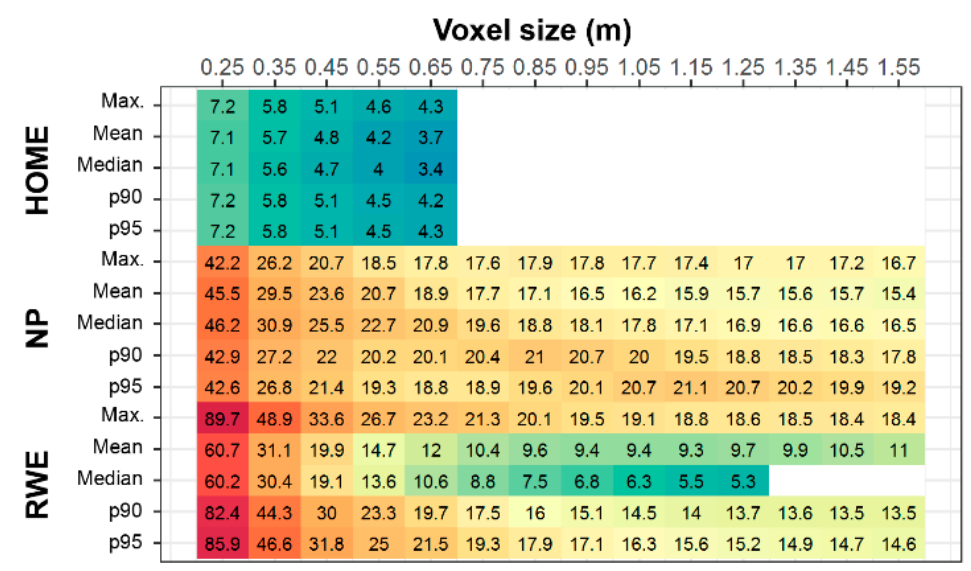

Figure 1. Average pulse density at which the corresponding ALSFw metric (HOME: height of median energy; NP: number of peaks; and RWE: return waveform energy) becomes stable for the different voxel sizes and assignation values. Smallest and largest values are represented by blue and red colors, respectively.

Table 1 shows the results from the estimation of understory vegetation variables through ALSFw. Similar results are found for the mean height and volume in terms of relative indicators (i.e., nRMSE and $\mathrm{CV}$ ) reaching a $\mathrm{R}^{2}$ of 0.949 and 0.951 , respectively, and a nRMSE of $7 \%$ for both.

Table 1. Estimate results ( $\mathrm{R}^{2}$ : coefficient of determination; RMSE: root mean square error; nRMSE: normalized RMSE; and CV: coefficient of variation) from the characterization of the understory vegetation.

\begin{tabular}{ccccc}
\hline Variable & $\mathbf{R}^{2}$ & RMSE & nRMSE & CV \\
\hline Mean height & 0.949 & $0.08 \mathrm{~m}$ & $7 \%$ & $11 \%$ \\
Volume & 0.951 & $56.49 \mathrm{~m}^{3}$ & $7 \%$ & $9 \%$ \\
\hline
\end{tabular}

\section{Discussion and Conclusions}

In this paper we assessed the variation of ALSFw metrics according to the influence of the pulse density and methodological parameters; and the potential of ALSF data for characterizing understory vegetation.

Overall, the results show that NP and RWE metrics are more unstable with a variable pulse density than HOME. While HOME become stable at SP values around 7 pulses $\mathrm{m}^{-2}$ with a voxel size 
of $0.25 \mathrm{~m}$, NP and RWE found SP values of 42-46 pulses $\mathrm{m}^{-2}$ and $60-90$ pulses $\mathrm{m}^{-2}$, respectively. However, the increment of the voxel size, and the use of the mean and median for the assignation value for RWE, make these two ALSFw more stable. Using the negative exponential distribution represents the steep increment of the metric values at low pulse densities, however the point where their values become stable is reached sooner (i.e., with a lower pulse density).

In addition, results also show the potential of ALSFw for characterizing the understory vegetation in Mediterranean forests. Estimation of mean height and volume are highly accurate, which shows the capacity of ALSFw to go further than ALS.

To know the point where ALSFw metrics become stable is essential for reducing side-lap effect. Pulse densities in a study area are not constant, hence this point provides the minimum pulse density at which the ALSFw metrics become stable. The selection of a minimum pulse density, given by the SP value, before planning an ALSFw acquisition or the selection of the most adequate methodological parameters for an existing data, reduce influence of the pulse density variation, and therefore sidelap effect. This leads to an improvement of the accuracy of the forest stand variables to be estimated.

Additionally, the accurate characterization of the understory vegetation from ALSFw data facilitates mapping this strata over large areas, which is essential for fire prevention.

Further studies must be addressed to assess the influence of the pulse density and ALSFW methodological parameters and to characterize understory vegetation in other ecosystems in order to draw more general conclusions about ALSFw data.

Acknowledgments: This study has been funded by the Spanish Ministerio de Economía y Competitividad and FEDER, in the framework of the project CGL2016-80705-R.

\section{References}

1. Marino, E.; Montes, F.; Tomé, J.L.; Navarro, J.A.; Hernando, C. Vertical forest structure analysis for wildfire prevention: Comparing airborne laser scanning data and stereoscopic hemispherical images. Int. J. Appl. Earth Obs. Geoinf. 2018, 73, 438-449, doi:10.1016/j.jag.2018.07.015.

2. Mallet, C.; Bretar, F. Full-waveform topographic lidar: State-of-the-art. ISPRS J. Photogramm. Remote Sens. 2009, 64, 1-16, doi:10.1016/j.isprsjprs.2008.09.007.

3. Crespo-Peremarch, P.; Ruiz, L.Á.; Balaguer-Beser, Á.; Estornell, J. Analyzing the role of pulse density and voxelization parameters on full-waveform LiDAR-derived metrics. ISPRS J. Photogramm. Remote Sens. 2018, 146, 453-464, doi:10.1016/j.isprsjprs.2018.10.012.

4. Crespo-Peremarch, P.; Tompalski, P.; Coops, N.C.; Ruiz, L.Á. Characterizing understory vegetation in Mediterranean forests using full-waveform airborne laser scanning data. Remote Sens. Environ. 2018, 217, 400-413, doi:10.1016/j.rse.2018.08.033.

5. Hancock, S.; Anderson, K.; Disney, M.; Gaston, K.J. Measurement of fine-spatial-resolution 3D vegetation structure with airborne waveform lidar: Calibration and validation with voxelised terrestrial lidar. Remote Sens. Environ. 2017, 188, 37-50, doi:10.1016/j.rse.2016.10.041.

6. Cao, L.; Coops, N.C.; Hermosilla, T.; Innes, J.; Dai, J.; She, G. Using small-footprint discrete and fullwaveform airborne LiDAR metrics to estimate total biomass and biomass components in subtropical forests. Remote Sens. 2014, 6, 7110-7135, doi:10.3390/rs6087110.

7. Akaike, H. Information theory and an extension of the maximum likelihood principle. In Proceedings of the 2nd International Symposium on Information; Petrow, B.N., Csaki, F., Ed.; Akademiai Kiado: Budapest, Hungary, 1973.

(C) 2019 by the authors. Submitted for possible open access publication under the terms and conditions of the Creative Commons Attribution (CC BY) license (http://creativecommons.org/licenses/by/4.0/). 